\title{
Higgs boson self-coupling measurements using ratios of cross sections
}

\section{Journal Article}

Author(s):

Goertz, Florian; Papaefstathiou, Andreas; Yang, Li L.; Zurita, José

Publication date:

2013-06

Permanent link:

https://doi.org/10.3929/ethz-b-000071000

Rights / license:

Creative Commons Attribution 4.0 International

Originally published in:

Journal of High Energy Physics 2013(6), https://doi.org/10.1007/JHEP06(2013)016 


\title{
Higgs boson self-coupling measurements using ratios of cross sections
}

\author{
Florian Goertz, ${ }^{b}$ Andreas Papaefstathiou, ${ }^{c}$ Li Lin Yang $^{a}$ and José Zurita ${ }^{d}$ \\ ${ }^{a}$ School of Physics and State Key Laboratory of Nuclear Physics and Technology, Peking University, \\ Beijing 100871, China \\ ${ }^{b}$ Institut für Theoretische Physik, ETH Zürich, \\ 8093 Zurich, Switzerland \\ ${ }^{c}$ Institut für Theoretische Physik, Universität Zürich, \\ 8057 Zurich, Switzerland \\ ${ }^{d}$ PRISMA Cluster of Excellence 8 \\ Mainz Institute for Theoretical Physics Johannes Gutenberg University, \\ 55099 Mainz, Germany \\ E-mail: fgoertz@phys.ethz.ch, andreasp@physik.uzh.ch, \\ yanglilin@pku.edu.cn, jose.zurita@uni-mainz.de
}

Abstract: We consider the ratio of cross sections of double-to-single Higgs boson production at the Large Hadron Collider at $14 \mathrm{TeV}$. Since both processes possess similar higherorder corrections, leading to a cancellation of uncertainties in the ratio, this observable is well-suited to constrain the trilinear Higgs boson self-coupling. We consider the scale variation, parton density function uncertainties and conservative estimates of experimental uncertainties, applied to the viable decay channels, to construct expected exclusion regions. We show that the trilinear self-coupling can be constrained to be positive with a $600 \mathrm{fb}^{-1}$ LHC dataset at $95 \%$ confidence level. Moreover, we demonstrate that we expect to obtain $\mathrm{a} \sim+30 \%$ and $\sim-20 \%$ uncertainty on the self-coupling at $3000 \mathrm{fb}^{-1}$ without statistical fitting of differential distributions. The present article outlines the most precise method of determination of the Higgs trilinear coupling to date.

Keywords: Higgs Physics, Standard Model

ARXiv EPRINT: 1301.3492 


\section{Contents}

1 Introduction 1

2 Dissection of the cross sections 2

3 Ratios of cross sections $\quad 4$

4 Constraining the self-coupling $\quad 7$

4.1 Variation with self-coupling and top quark Yukawa $\quad 7$

4.2 Assumptions for experimental uncertainties 8

$\begin{array}{ll}4.3 & \text { Deriving constraints }\end{array}$

$\begin{array}{llr}5 & \text { Conclusions } & 14\end{array}$

\section{Introduction}

One of the aims of the Large Hadron Collider (LHC) is to search for the agent of electroweak symmetry breaking (EWSB), which in its minimal form is the Standard Model (SM) Higgs boson $(H)$. Recently, both the ATLAS and the CMS collaborations have observed a new state with a mass of about $125 \mathrm{GeV}$, whose properties are in substantial agreement with the SM Higgs boson [1-5]. The quest for understanding the mechanism behind EWSB does not end with the discovery of this particle. It is crucial to test the Higgs sector to its full extent, measuring the couplings of the Higgs boson to gauge bosons and matter fields [630], and also to probe its self-interactions [31-36]. After EWSB, the Higgs potential can be written as

$$
V(H)=\frac{1}{2} M_{H}^{2} H^{2}+\lambda_{H H H} v H^{3}+\frac{1}{4} \lambda_{H H H H} H^{4} .
$$

In the SM, $\lambda_{H H H}^{\mathrm{SM}}=\lambda_{H H H H}^{\mathrm{SM}}=\left(M_{H}^{2} / 2 v^{2}\right) \approx 0.13$ for a Higgs mass of $M_{H} \simeq 125 \mathrm{GeV}$ and a vacuum expectation value of $v \simeq 246 \mathrm{GeV}$. We can also define normalised couplings $\lambda \equiv \lambda_{H H H} / \lambda_{H H H}^{\mathrm{SM}}$ and $\tilde{\lambda} \equiv \lambda_{H H H H} / \lambda_{H H H H}^{\mathrm{SM}}$.

A measurement of these two couplings is crucial to the reconstruction of the Higgs potential and will allow testing of the EWSB mechanism. Moreover, in many models beyond the SM, these couplings may deviate from the SM values, and in that case they will provide relevant information about the nature of the new physics model.

At the LHC, the quartic coupling $\tilde{\lambda}$ may be probed via triple Higgs boson production. However, its tiny cross section [37] makes it very difficult, if not impossible, to do so. On the other hand, the trilinear coupling $\lambda$ can be measured in Higgs boson pair production, $p p \rightarrow H H$, which may be discovered at a large luminosity phase of the LHC.

The discovery potential for Higgs boson pair production at the LHC has been studied in $[32-35,38]$. In refs. [32, 38], constraints were placed on $\lambda$ using statistical fits to 
the shape of the visible mass distributions of the final decay products of the Higgs pairs, whereas refs. $[33,34]$ focused on the establishment of the Higgs pair production process using improved techniques originating mainly from developments in the understanding of boosted jet substructure [39, 40]. In ref. [35] the final state $b \bar{b} \gamma \gamma$ was revisited as well as $b \bar{b} \tau^{+} \tau^{-}$and $b \bar{b} W^{+} W^{-}$(fully leptonic), without making use of jet substructure techniques (although boosted Higgs bosons were required). The present article concentrates on using the results from the available phenomenological studies along with the best available theoretical cross section calculations and conservative estimates of the experimental uncertainties, to demonstrate the possibility of constraining the trilinear Higgs self-coupling at the LHC.

The article is organised in the following way: in section 2 we dissect the Higgs boson production cross sections and in section 3 we examine the theoretical uncertainties on the ratio of cross sections of double-to-single Higgs production. Then, in section 4, we present the expected constraints obtained at integrated luminosities of $600 \mathrm{fb}^{-1}$ and $3000 \mathrm{fb}^{-1}$ for a simplified model, as well as within the Standard Model. We conclude in section 5 .

\section{Dissection of the cross sections}

The Higgs boson pair production cross section is dominated by gluon fusion, as is the single production cross section [41, 42]. For the pair production, other modes, like $q q \rightarrow q q H H, V H H, t \bar{t} H H$ are a factor of 10-30 smaller [35, 36, 43, 44], and thus we do not consider them in the rest of our analysis. At leading order (LO), there are two main contributions: a diagram containing a 'triangle' loop, and one containing a 'box' loop of heavy quarks, as shown in figure 1. By far the most dominant contribution comes from the top quark loops, with a smaller sub-dominant bottom quark contribution. The production of a single, on-shell Higgs boson only contains a diagram of the 'triangle' type. The triangle diagram can only contain initial-state gluons in a spin-0 state, whereas the box contribution can contain both spin-0 and spin-2 configurations. Therefore, there are two Lorentz structures involved in the box diagram matrix element. At LO, we may write, schematically:

$$
\sigma_{H H}^{\mathrm{LO}}=\left|\sum_{q}\left(\alpha_{q} C_{q, \text { tri }}^{(1)}+\beta_{q} C_{q, \text { box }}^{(1)}\right)\right|^{2}+\left|\sum_{q} \gamma_{q} C_{q, \text { box }}^{(2)}\right|^{2}
$$

where $C_{q, \text { tri }}^{(1)}$ represents the matrix element for the triangle contributions and $C_{q, \text { box }}^{(i)}$ represents the matrix element for the two Lorentz structures $(i=1,2)$ coming from the box contributions [41, 45], for each of the quark flavours $q=\{t, b\}$.

The parameters $\alpha_{q}, \beta_{q}$ and $\gamma_{q}$ for quark flavour $q$ are given in terms of the Standard Model Lagrangian parameters by:

$$
\begin{aligned}
\alpha_{q} & =\lambda y_{q}, \\
\beta_{q} & =\gamma_{q}=y_{q}^{2},
\end{aligned}
$$

where $q=\{t, b\}, \lambda$ is the (normalised) Higgs triple coupling defined in the previous section and $y_{q}$ is the normalised $H q \bar{q}$ coupling (after electroweak symmetry breaking and 



Figure 1. The Higgs pair production diagrams contributing to the gluon fusion process at LO are shown, for a generic fermion $f$.

assumed to be real) defined with respect to the SM value: $y_{q} \equiv Y_{q} / Y_{q}^{\mathrm{SM}}$ ( $Y_{q}$ being the resulting coupling and $Y_{q}^{\mathrm{SM}}$ the $\mathrm{SM}$ value). In contrast, the single Higgs cross section, again, schematically, will only contain the matrix element squared $\left|\sum_{q} C_{q, \text { tri }}^{(1)}\right|^{2}$.

We have performed numerical fits using the results of the hpair program [46], used to calculate the total cross section for Higgs boson pair production at leading and approximate next-to-leading (NLO) orders. The fits were done employing MSTW2008lo68cl and MSTW2008nlo68cl parton density functions [47] and using top and bottom quark masses of $174.0 \mathrm{GeV}$ and $4.5 \mathrm{GeV}$ respectively. We have obtained:

$$
\begin{aligned}
\sigma_{H H}^{\mathrm{LO}}[\mathrm{fb}] & =5.22 \lambda^{2} y_{t}^{2}-25.1 \lambda y_{t}^{3}+37.3 y_{t}^{4}+\mathcal{O}\left(\lambda Y_{b} y_{t}^{2}\right), \\
\sigma_{H H}^{\mathrm{NLO}}[\mathrm{fb}] & =9.66 \lambda^{2} y_{t}^{2}-46.9 \lambda y_{t}^{3}+70.1 y_{t}^{4}+\mathcal{O}\left(\lambda Y_{b} y_{t}^{2}\right),
\end{aligned}
$$

where we are not showing terms suppressed by the (un-normalised) $H b \bar{b}$ coupling, $Y_{b}$. In fact, we have checked explicitly that a fit performed ignoring the bottom quark terms results in form factors accurate at the $1 \%$ level and a total cross section accurate to better than the $0.2 \%$ level (within the SM). Thus, for simplicity, we neglect the bottom contributions in the discussion that follows in the rest of this section. We do, however, include the bottom quark loops in our numerical results throughout this paper.

It is evident from eqs. (2.1)-(2.3) that the Higgs pair production cross section contains an interference term proportional to $\left(\lambda y_{t}^{3}\right)$. Hence, for positive values of $\left(\lambda y_{t}^{3}\right)$ the cross section is reduced, whereas for negative values, it is enhanced. The box squared term is dominant, and scales as $y_{t}^{4}$, whereas the triangle squared term is subdominant due to the off-shell Higgs boson which then decays to Higgs boson pairs, and scales as $\lambda^{2} y_{t}^{2}$. Also note that there exists a minimum value of $\sigma_{H H}^{\mathrm{NLO}}$ at $\lambda=\lambda_{\min } \simeq 2.46 y_{t}$ (taking into account the bottom quark contributions). The cross section $\sigma_{H H}$ is symmetric about the point $\lambda_{\min }$.

We note that the above structure, and hence the different contributions to the cross section, can of course be modified if new physics that allows new resonances to run in the triangle and box loops (or adds new couplings, like an $f f H H$ interaction) is present [4853]. For simplicity, in the present article we will focus on the Standard Model itself, as well as scenarios where the possible higher-dimensional operators, induced by such new physics, are subdominant with respect to changes in the $\lambda$ and $y_{t}$ couplings.

Examples of such scenarios would be models where a Higgs boson $H$ mixes with another scalar $S$, like in Higgs Portal [54, 55] or Two-Higgs Doublets Models (see, e.g. [56]), where 
no new particles run in the loop. Here the pair production cross section of the SM-like Higgs boson $H$ will get modified only by having a resonant effect in the s-channel diagram, due to the new scalar. ${ }^{1}$ Indeed, one can obtain a $10-20 \%$ change in $y_{t}$ and arbitrary values for $\lambda$, together with a negligible resonant contribution, by selecting appropriately the free parameters that appear in such theories. ${ }^{2}$ The new scalar $S$ may be outside of LHC reach if it is sufficiently heavy, or with reduced couplings to SM particles (see, e.g. [57]). Even if the new scalar particle is observed, the measurement of the parameter $\lambda$ will still be a meaningful and interesting question.

\section{Ratios of cross sections}

It has been pointed out in ref. [26] that the ratio of cross sections between Higgs pair production and single Higgs production:

$$
C_{H H}=\frac{\sigma(g g \rightarrow H H)}{\sigma(g g \rightarrow H)} \equiv \frac{\sigma_{H H}}{\sigma_{H}},
$$

could be more accurately determined theoretically than the Higgs-pair production cross section itself. ${ }^{3}$ This is based on the fact that the processes are both gluon-initiated and the respective higher-order QCD corrections could be very similar. Hence, it is assumed that a large component of the QCD uncertainties drop out in the ratio $C_{H H}$. Moreover, experimental systematic uncertainties that affect both cross sections may cancel out by taking the ratio. An example is the luminosity uncertainty, which should cancel out provided the same amount of data is used in both measurements.

Here we investigate the extent to which the above assumptions are correct, using the available calculations for the cross sections. We begin by considering the LO and NLO calculations for $\sigma(g g \rightarrow H H)$ and $\sigma(g g \rightarrow H)$ at the LHC at $14 \mathrm{TeV} \cdot{ }^{4}$ Using the MSTW2008lo68cl and MSTW2008nlo68cl parton density functions [47], we show in figures 2 and 3 the cross sections as well as their ratios, $C_{H H}$, as a function of the Higgs mass at both LO and NLO. ${ }^{5}$ We present the scale uncertainty obtained by varying the factorisation and renormalization scales (set to be equal) between $\left[0.5 \mu_{0}, 2.0 \mu_{0}\right]$, where $\mu_{0}=M_{H}$

\footnotetext{
${ }^{1}$ Even if new coloured fermions are present, their contribution can be neglected if their couplings are small or if they are very heavy and decouple.

${ }^{2}$ In specific examples we have found that one can arrange to have a heavy $S$ particle with a small $S H H$ coupling, such that its resonance effect will not affect the SM-like Higgs pair production rate, and with a moderate deviation in the respective $H H H$ coupling. The price to pay for $S$ being heavy is to have the other trilinear scalar couplings, $S S H$ and $S S S$ to be $\mathcal{O}(1)$, but still consistent with the perturbativity condition, $\lambda \ll \sqrt{4 \pi}$.

${ }^{3}$ Note that a somewhat different, but related, idea of taking ratios of cross sections for various processes at different energies was explored in [58].

${ }^{4}$ All calculations in the present section have been performed in the SM, i.e. $\lambda=1$ and $y_{t}=1$. We do not expect the theoretical uncertainties to vary substantially with these values, since the variation arises from terms with logarithmic ratios of scales, whose coefficients are often determined by universal QCD functions, namely the $\beta$ function or the Altarelli-Parisi kernels, depending on whether the renormalization or factorization scale is involved.

${ }^{5}$ It is important to note that the NLO calculation for $H H$ production has been performed in the heavy top mass limit, and hence it is expected to be approximate. At LO, the accuracy of the large top mass approximation is $\mathcal{O}(10 \%)[31,52,59]$. Note that the sub-dominant effects of the bottom quark are kept in
} 

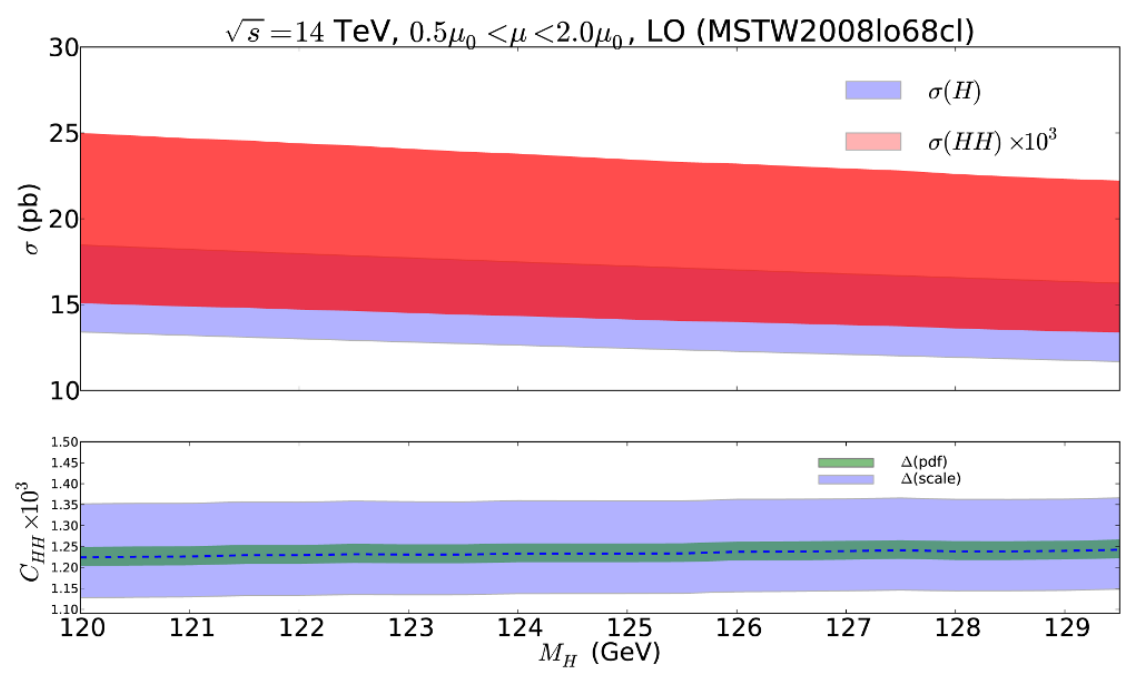

Figure 2. The cross sections for single and double Higgs boson production at leading order using the MSTW2008lo68cl PDF set. In the lower plot, the fractional uncertainty due to scale variation is shown in the blue band, as well as the PDF uncertainty in the green band.

for the higlu program, used to obtain the single Higgs cross sections [60], and $\mu_{0}=M_{H H}$ for the hpair program (where $M_{H H}$ is the invariant mass of the Higgs pair), used for the Higgs pair production cross sections [46]. The scale choices are the natural ones for each of the processes but we verified that the conclusions are not altered substantially by changing the hpair scale, i.e. the numerator, to equal the scale that appears in the denominator, $\mu_{0}=M_{H}$. Implicit in the calculation of the scale uncertainty of the ratio $C_{H H}$, is the fact that the scale variation of the single and double Higgs cross sections between $0.5 \mu_{0}$ and $2.0 \mu_{0}$ is fully correlated: i.e., we obtain the upper and lower variations of the ratio by dividing the cross sections with the same magnitude of variation of the scale. This is an approximation that is justified since the two processes possess similar topologies, and is in fact one of the main insights in favour of using $C_{H H}$. We also show, in the ratio, the resulting PDF uncertainty, calculated using the MSTW2008nlo68cl error sets according to the prescription found in [61].

Several observations on the behaviour of the $C_{H H}$ ratio can be made. First of all, it is evident that the fractional uncertainty due to scale variation is reduced with respect to the individual calculations in both leading and next-to-leading orders: for the LO case, the individual cross sections have a $\sim \pm 20 \%$ (single Higgs boson production) and $\sim \pm 25 \%$ (double Higgs boson production) scale uncertainty, whereas the ratio has a $\sim \pm 9 \%$ scale uncertainty. For the NLO case, it is reduced from $\sim \pm 17 \%$ (single and double Higgs boson production) to $\sim \pm 1.5 \%$ for the ratio. ${ }^{6}$

the calculations throughout the paper where they are available: up to LO in $H H$ production and to NLO in single Higgs production.

${ }^{6}$ Note that in ref. [62], threshold resummation effects in SM Higgs pair production in soft-collinear effective theory were considered. The authors claim a reduction of the scale uncertainty to $3 \%$. For other resummation studies in single Higgs production see, for example [63-65]. 

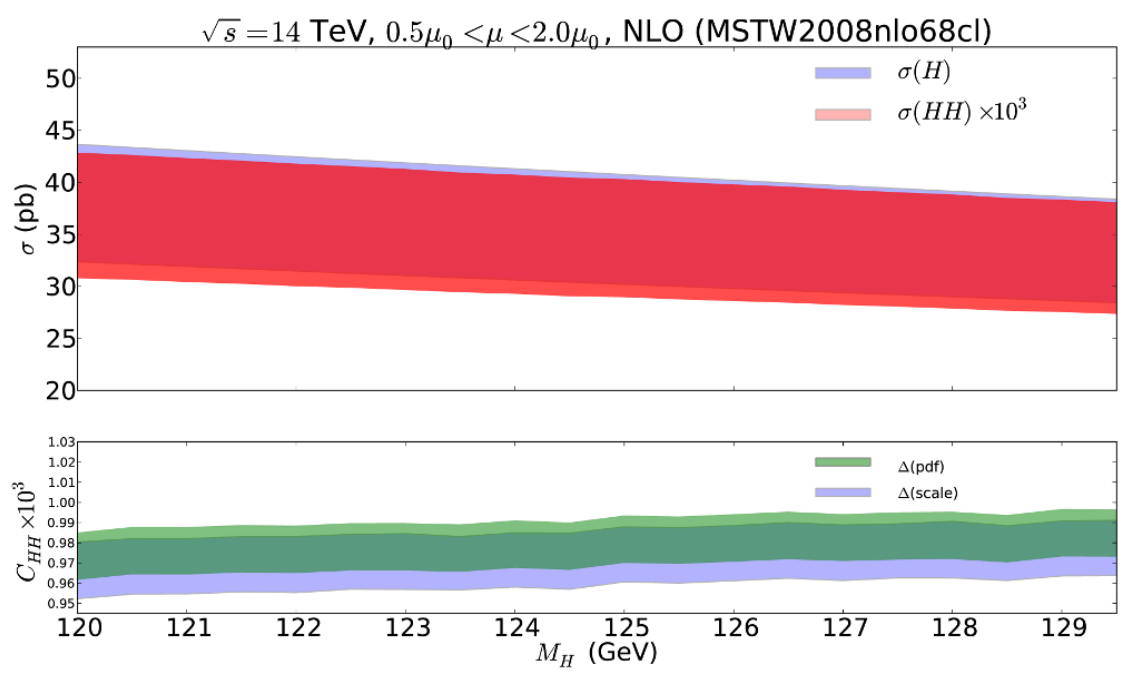

Figure 3. The cross sections for single and double Higgs boson production at next-to-leading order using the MSTW2008nlo68cl PDF set. In the lower plot, the fractional uncertainty due to scale variation is shown in the blue band, as well as the PDF uncertainty in the green band.

Furthermore, we can explicitly see that the uncertainty due to the QCD corrections partially cancels out: even though the individual K-factors in the cross sections $\sigma_{H}$ and $\sigma_{H H}$ are large, they are also very similar, both being $\sim 2$. As a consequence, the central value of the ratio only decreases by a small amount from $\sim 1.25$ to $\sim 1.0$ when going from LO to NLO. This is an indication that higher order corrections are quite likely to change the ratio by an even smaller fraction than the change from LO to NLO, when it is considered at NNLO, whereas the single Higgs production cross section has a K-factor of about $\sim 1.5$ when compared to the NLO calculation [66]. ${ }^{7}$ These findings support the idea of employing the fully correlated scale variation described before as a realistic estimate for the theoretical error. ${ }^{8}$

The PDF uncertainties for the cross sections themselves are not shown since they are of the order of a few $\%$ and hence subdominant. The PDF uncertainty is also subdominant in the case of the $\mathrm{LO}$ ratio, as shown in figure 2. In the case of the NLO ratio, the PDF uncertainty becomes comparable to the scale uncertainty as can be seen in figure 3. Combining the two errors in quadrature would induce an error of $\pm \mathcal{O}(3 \%)$, still smaller than the $\sim \pm 17 \%$ error on the NLO Higgs pair production cross section. To remain conservative, we will assume that the theoretical errors on $C_{H H}$ and $\sigma_{H H}$ are $\pm 5 \%$ and $\pm 20 \%$, respectively, in what follows.

\footnotetext{
${ }^{7}$ An equivalent calculation at NNLO does not presently exist for Higgs pair production.

${ }^{8}$ Note that studies of theoretical uncertainties in single and double Higgs production can be found, respectively, in refs. [35, 67].
} 


\section{Constraining the self-coupling}

In the studies conducted in refs. [32, 38], the Higgs self-coupling was constrained using the final states $b \bar{b} \gamma \gamma, b \bar{b} \mu^{+} \mu^{-}$and $W^{+} W^{-} W^{+} W^{-}$(in the high Higgs mass region). The constraints were obtained by fitting the visible mass distributions in each process for the signal and backgrounds.

Here we choose to follow a different strategy: taking into account the facts that the different signal channels possess a relatively low number of events and that the shapes of distributions for the backgrounds (and even the signal) are not always very well known, we employ only information originating from the rates. Furthermore, we use the theoretically more stable ratio $C_{H H}$ between the double and single Higgs production cross sections, examined in the previous section. We focus on luminosities of $600 \mathrm{fb}^{-1}$ and $3000 \mathrm{fb}^{-1}$ that can be respectively obtained by ATLAS and CMS together in the first long-term $14 \mathrm{TeV}$ run, or by the individual experiments in an even longer-term run at the same energy. We do not attempt to combine between the individual channels, as this will require a more detailed study from the experimental collaborations.

\subsection{Variation with self-coupling and top quark Yukawa}

To quantify the possible region that can be constrained using the ratio $C_{H H}$, we first examine the behaviour of the cross section for Higgs pair production and the ratio $C_{H H}$ at $14 \mathrm{TeV}$, when varying the self-coupling $\lambda$, as well as the top Yukawa, $y_{t}$. It is important to consider the variation of the top quark Yukawa determination, since the production rates of both double and single Higgs production can be substantially affected. Moreover, the expected accuracy on the top quark Yukawa is expected to be $\pm \mathcal{O}(15 \%)$ at $300 \mathrm{fb}^{-1}$ of LHC data at $14 \mathrm{TeV}$ [68].

We show the cross section $\sigma_{H H}$ and ratio $C_{H H}$ at $y_{t}=1$ as a function of $\lambda$, as well as both quantities at $\lambda=1$ as a function of $y_{t}$ in figures 4 and 5, respectively. Evidently, the effects of both $\lambda$ and $y_{t}$ are significant: the cross section varies from $\sim 30 \mathrm{fb}$ at $\left(\lambda, y_{t}\right)=(1,1)$ (i.e. the $\mathrm{SM}$ values) to $\sim 125 \mathrm{fb}$ at $\left(\lambda, y_{t}\right)=(-1,1)$ and $\sim 300 \mathrm{fb}$ at $\left(\lambda, y_{t}\right)=(1,1.6)$. The ratio itself varies from $\sim 10^{-3}$ at $\left(\lambda, y_{t}\right)=(1,1)$ to $\sim 3.5 \times 10^{-3}$ at $\left(\lambda, y_{t}\right)=(-1,1)$ and $\left(\lambda, y_{t}\right)=(1,1.6)$. It is obvious that negative values of $\lambda$ can be excluded sooner than the positive values, since the cross section and ratio of cross sections both increase fast with decreasing $\lambda$.

We note that negative values of $y_{t}$ are currently viable [21] and physical, and could arise in beyond-the-SM physics models. Since Higgs pair production only depends on the sign of the product $\left(\lambda y_{t}\right)$, the corresponding values for $y_{t}<0, \lambda>0$ are equivalent to those for the points with the same absolute values of the parameters but $y_{t}>0, \lambda<0 .{ }^{9}$

\footnotetext{
${ }^{9}$ Note that the degeneracy with respect to the sign of $y_{t}$ that appears in Higgs pair production may be resolved through the study of different processes long before the Higgs self-coupling is probed. See, for example, refs. [69, 70].
} 

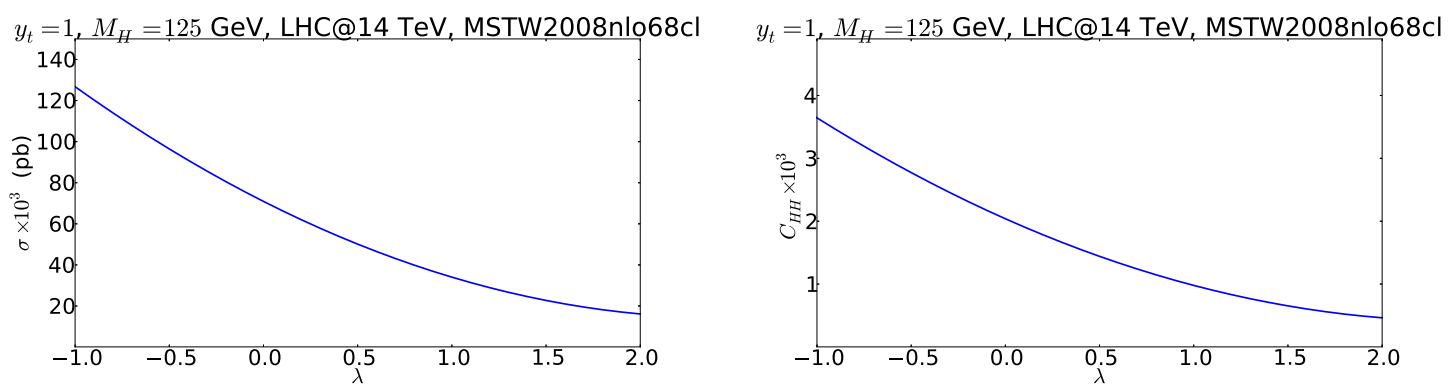

Figure 4. The cross section for double Higgs production and the ratio $C_{H H}$ at next-to-leading order using the MSTW2008nlo68cl PDF set, as a function of $\lambda$ at $y_{t}=1$.
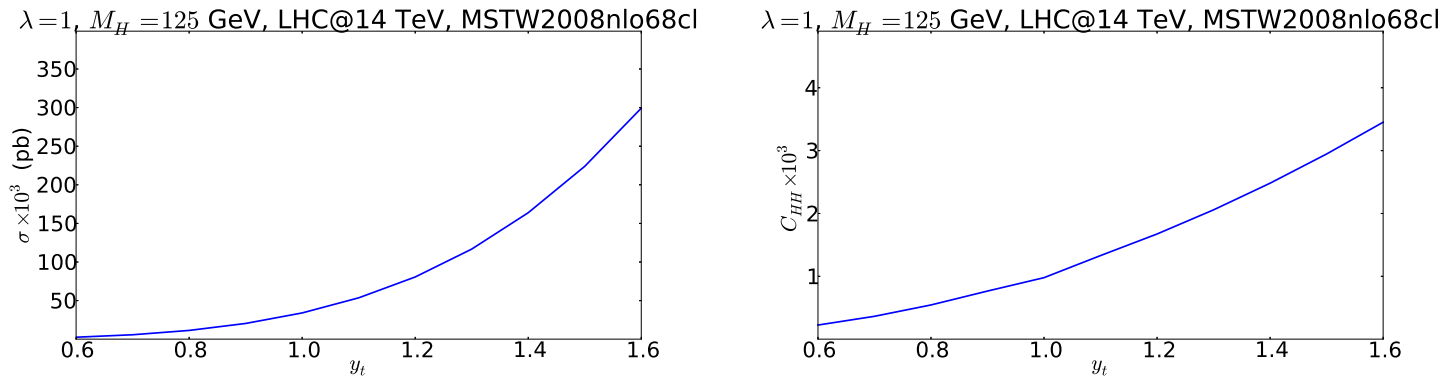

Figure 5. The cross section for double Higgs production and the ratio $C_{H H}$ at next-to-leading order using the MSTW2008nlo68cl PDF set, as a function of $y_{t}$ at $\lambda=1$.

\subsection{Assumptions for experimental uncertainties}

The ratio $C_{H H}$ can be used to derive the expected constraints that can be obtained at a $14 \mathrm{TeV}$ LHC for different physics models, including the SM. Certain assumptions on the systematic uncertainties need to be made for the branching ratios related to each mode. We first define the following quantities:

$$
\begin{aligned}
\sigma_{H H}^{b \bar{b} x x} & \equiv 2 \times \sigma_{H H} \times \operatorname{BR}(b \bar{b}) \times \operatorname{BR}(x x), \\
\sigma_{H}^{b \bar{b}} & \equiv \sigma_{H} \times \operatorname{BR}(b \bar{b}),
\end{aligned}
$$

where $x x$ denotes the $H \rightarrow x x$ decay mode in question. Hence, we can invert the above relations to obtain:

$$
C_{H H}^{\exp .}=\left.\frac{\sigma_{H H}^{b \bar{b} x x}}{\sigma_{H}^{b \bar{b}} \times B R(x x)}\right|_{\text {exp. }},
$$

which is the experimental measurement of the theoretical quantity $C_{H H}$.

Since the scope of this article is not a detailed experimental study, we now make several assumptions on the measurement uncertainties for each of the quantities in the ratio of eq. (4.2). We focus on the region $\lambda \in(-1.0, \sim 2.46)$, since the cross section is symmetric with respect to the minimum at $\lambda \simeq 2.46$. According to ref. [71], the branching ratio of $H \rightarrow b \bar{b}$ times the cross section for single Higgs is expected to be known to $\pm 20 \%$ after $300 \mathrm{fb}^{-1}$ of data at $14 \mathrm{TeV}$, and hence we assume that the uncertainty on $\sigma_{H}^{b \bar{b}}$ is $\pm 20 \%$. 
Similarly, according to [71], the uncertainties on $\operatorname{BR}\left(\tau^{+} \tau^{-}\right), \operatorname{BR}\left(W^{+} W^{-}\right)$and $\operatorname{BR}(\gamma \gamma)$ are expected to be $\pm 12 \%, \pm 12 \%$ and $\pm 16 \%$, respectively, at $300 \mathrm{fb}^{-1}$. To remain conservative, we assume that going beyond $300 \mathrm{fb}^{-1}$ of luminosity, there will be no improvement on these uncertainties. This can be true, for example, if the measurements are dominated by systematic uncertainties that cannot be improved further. Moreover, the uncertainty on the cross section of the measured final state, $\Delta \sigma_{H H}^{b \bar{b} x x}$, is estimated by assuming that the Poisson distribution of the obtained number of events can be approximated by a Gaussian, for simplicity. Hence, if we expect a number of $B$ background events and we experimentally measure $N$ events, the error on the signal estimate, $S=N-B$, is given by $\Delta S=\sqrt{N+B}$. The expected number of events for the studies we consider below were taken from [33, 34, 38]. We combine all the estimates of the uncertainties in quadrature for each mode to obtain an estimate of the total uncertainty:

$$
\left(\frac{\Delta C_{H H}}{C_{H H}}\right)^{2}=\left(\frac{\Delta \sigma_{H H}^{b \bar{b} x x}}{\sigma_{H H}^{b \bar{b} x x}}\right)^{2}+\left(\frac{\Delta \mathrm{BR}(x x)}{\operatorname{BR}(x x)}\right)^{2}+\left(\frac{\Delta \sigma_{H}^{b \bar{b}}}{\sigma_{H}^{b \bar{b}}}\right)^{2} .
$$

In what follows we also add the theoretical error estimates in quadrature to the above.

\subsection{Deriving constraints}

The ratio of cross sections considered in section 3 was calculated under the assumption of validity of the SM. In general, if one wishes to use the ratio to perform a study of a different model with a given set of parameters $\left\{p_{i}\right\}$, one should first:

- Calculate the ratio $C_{H H}$ and the corresponding theoretical error as a function of the set of parameters $\left\{p_{i}\right\}$. The set $\left\{p_{i}\right\}$ may, for example, include the new masses and couplings of the theory or coefficients of new higher-dimensional operators.

- Estimate, as well as possible, the expected experimental errors arising from the measurements of the different components that comprise the experimental value of the ratio $C_{H H}^{\exp }$, as we have done in the previous section.

With the above at hand, one can then form the following question:

Given an assumption for the 'true' value of a subset of the model parameters, what is the constraint we expect to impose on these parameters through Higgs pair production?

Following the above framework, here we perform a study of a simplified model, which we present as an example of an implementation of the above steps. Thus, we consider a situation where the Standard Model is valid almost everywhere, except that we allow the variation of the parameters $\left\{p_{i}\right\}=\left\{\lambda, y_{t}\right\}$. As we have already discussed at the end of section 2, such situations may arise in Higgs Portal or Two-Higgs Doublets Models. Furthermore, in the same framework, this simplified model will also provide us with limits on the determination of $\lambda$ within the SM, by setting the 'true' values of $\lambda$ and $y_{t}, \lambda_{\text {true }}=1=y_{t, \text { true }}$. 


\begin{tabular}{|l|c|c|c|}
\hline Process & $\mathrm{S} / \mathrm{B}\left(600 \mathrm{fb}^{-1}\right)$ & $\Delta C_{H H} / C_{H H}\left(600 \mathrm{fb}^{-1}\right)$ & $\Delta C_{H H} / C_{H H}\left(3000 \mathrm{fb}^{-1}\right)$ \\
\hline$b \bar{b} \tau^{+} \tau^{-}$ & $50 / 104$ & 0.400 & 0.279 \\
\hline$b \bar{b} W^{+} W^{-}$ & $11.2 / 7.4$ & 0.513 & 0.314 \\
\hline$b \bar{b} \gamma \gamma$ & $6 / 12.5$ & 0.964 & 0.490 \\
\hline
\end{tabular}

Table 1. The table shows expected number of signal (S) and background (B) events for SM Higgs pair production, resulting at $600 \mathrm{fb}^{-1}$, and the respective fractional uncertainties on the ratio of double-to-single Higgs boson production cross sections, $\Delta C_{H H} / C_{H H}$, for the different channels and the two investigated LHC luminosities, $600 \mathrm{fb}^{-1}$ and $3000 \mathrm{fb}^{-1}$, using $M_{H}=125 \mathrm{GeV}$. The fractional uncertainties include the theoretical error due to the scale/parton density functions uncertainties, assumed to be $5 \%$.

We start by fixing the value of the top Yukawa in this simplified model to be $y_{t}=y_{t, \text { true }}=1$. Thus, to answer to the above question we produce an 'exclusion' plot, calculated by drawing the curves that result in expected measurements that are one or two standard deviations away from the central value of $C_{H H}$, which is assumed to be equal to that given by $\lambda_{\text {true }}$. By virtue of this definition, it is obvious that the central value itself is, of course, not expected to be excluded. Equivalent plots in this model can be constructed, by fixing $\lambda_{\text {true }}$ and varying $y_{t, \text { true }}$, but we do not perform these here.

Using $C_{H H}$ we draw such curves for $600 \mathrm{fb}^{-1}$ of data in figures 6,7 and 8 for the final states $b \bar{b} \tau^{+} \tau^{-}, b \bar{b} W^{+} W^{-}$and $b \bar{b} \gamma \gamma$, respectively. To bring the three channels to an equal footing, we have rescaled the $b \bar{b} \tau^{+} \tau^{-}$cross section in [33] by employing a factor of $32.4 / 28.4$ accounting for the central value of the NLO production cross section used in [34], and moreover, rescaled by $0.7^{2} / 0.8^{2}$ for a reduced $\tau$-jet tagging efficiency. For the $b \bar{b} W^{+} W^{-}$ mode in [34] we also include the tauonic decays of the $W$ bosons, and for the $b \bar{b} \gamma \gamma$ result in [38] we average between the 'hi' and 'lo' LHC results to get 6 versus 12.5 events at $600 \mathrm{fb}^{-1} .{ }^{10}$ We have not rescaled the $b \bar{b} \gamma \gamma$ analysis, since this was done for a Higgs of mass $120 \mathrm{GeV}$ in [38]. In the lower panel of figure 6 we also show the exclusion regions extracted by using the Higgs pair production cross section measurement itself, with an associated uncertainty of $\pm 20 \%$. We assume that the uncertainty on $\operatorname{BR}(b \bar{b})$ is the same as that on $\sigma_{H}^{b \bar{b}}$, namely $\pm 20 \%$. It is obvious that the exclusion obtained from the cross section is expected to be weaker than that obtained by the ratio, due to the larger theoretical systematic uncertainty on the cross section itself. Moreover, the expected exclusion from $\sigma_{H H}$ will be more affected by experimental systematic uncertainties which would add to the errors. For completeness, we show the estimated fractional uncertainty on the ratio, $\Delta C_{H H} / C_{H H}$, used to extract the exclusion regions, for the different processes and investigated luminosities in table 1. At high luminosity the uncertainties all tend to similar numbers since we have assumed that the other contributing uncertainties $\left(\Delta \mathrm{BR}(x x)\right.$ and $\left.\Delta \sigma_{H}^{b \bar{b}}\right)$ do not improve and they become systematic-dominated. These values are provided for completeness, as an indication, and merit further investigation by the experimental collaborations.

\footnotetext{
${ }^{10}$ The 'hi' and 'lo' refer to, respectively, the conservative and optimistic assumptions made in [38] for the jet to photon misidentification probability.
} 


\begin{tabular}{|l|c|c|c|c|}
\hline Process & $600 \mathrm{fb}^{-1}(2 \sigma)$ & $600 \mathrm{fb}^{-1}(1 \sigma)$ & $3000 \mathrm{fb}^{-1} 2 \sigma$ & $3000 \mathrm{fb}^{-1} 1 \sigma$ \\
\hline$b \bar{b} \tau^{+} \tau^{-}$ & $(0.22,4.70)$ & $(0.57,1.64)$ & $(0.42,2.13)$ & $(0.69,1.40)$ \\
\hline$b \bar{b} W^{+} W^{-}$ & $(0.04,4.88)$ & $(0.46,1.95)$ & $(0.36,4.56)$ & $(0.65,1.46)$ \\
\hline$b \bar{b} \gamma \gamma$ & $(-0.56,5.48)$ & $(0.09,4.83)$ & $(0.08,4.84)$ & $(0.48,1.87)$ \\
\hline
\end{tabular}

Table 2. The expected limits on $\lambda$ at $1 \sigma$ and $2 \sigma$ confidence levels in the Standard Model $\left(\lambda_{\text {true }}=1\right.$, $\left.y_{t, \text { true }}=1\right)$. The results have been derived using $C_{H H}$ and are shown for $600 \mathrm{fb}^{-1}$ and $3000 \mathrm{fb}^{-1}$. Note that there can be either one or two regions, in both cases symmetric about the minimum at $\lambda \simeq 2.46$. Where there may exist a second valid region, we only show the lower one.

The interpretation of the 'exclusion' curves is simple: as an example, if we assume or believe that the 'true' value of the triple Higgs coupling in this model is $\lambda_{\text {true }}=1$, then by examining figure 6 for the $b \bar{b} \tau^{+} \tau^{-}$mode at $600 \mathrm{fb}^{-1}$, we can conclude that using $C_{H H}$ the expected experimental result should lie within $\lambda \in(0.57,1.64)$ with $\sim 68 \%$ confidence level. We expect to exclude any values outside this range after $600 \mathrm{fb}^{-1}$, given the value $\lambda_{\text {true }}=1$. We show the collected exclusion limits for $\lambda_{\text {true }}=1$ and $y_{t, \text { true }}=1$ (i.e. the SM values) at $1 \sigma$ and $2 \sigma$ at $600 \mathrm{fb}^{-1}$ as well as the end-of-run LHC integrated luminosity of $3000 \mathrm{fb}^{-1}$ in table 2. The $3000 \mathrm{fb}^{-1}$ values have also been calculated by assuming no improvement in the uncertainty estimates that we have assumed at $600 \mathrm{fb}^{-1}$. The table demonstrates an important conclusion: it is possible, using the discovery of the three viable channels, to constrain the trilinear coupling $\lambda$ in the SM to be positive at $95 \%$ confidence level at $600 \mathrm{fb}^{-1}$. Moreover, a naive combination of the 'uncertainties', at $1 \sigma$ about $\lambda_{\text {true }}$, over the three channels indicates that a measurement of accuracy $\sim+30 \%$ and $\sim-20 \%$ is possible simply by using the rates at $3000 \mathrm{fb}^{-1}$. Note that the curves have been drawn up to $\lambda_{\min } \simeq 2.46$. The regions beyond that value are determined by the mirror symmetry with respect to $\lambda_{\min }$ (the cross section is degenerate for $\lambda \rightarrow 2 \lambda_{\min }-\lambda$, which makes those values of $\lambda$ indistinguishable).

We should emphasise at this point that figures 6, 7 and 8 do not represent the Standard Model, except at $\lambda_{\text {true }}=1$, and should be taken simply as an example of the suggested framework in a simplified, but still not unrealistic, scenario.

It is interesting to compare the regions obtained by the above method for the SM, with those obtained in ref. [38], where the authors used the only viable mode for a low mass Higgs boson at the time $\left(M_{H}=120 \mathrm{GeV}\right), b \bar{b} \gamma \gamma$, to extract $\lambda$ from the visible mass distribution. After background subtraction, their best limit at $600 \mathrm{fb}^{-1}$ was $\lambda \in(0.26,1.94)$ at $1 \sigma$. Here, for the $b \bar{b} \tau^{+} \tau^{-}$we obtain $\lambda \in(0.57,1.64)$, for the $b \bar{b} W^{+} W^{-}$mode we obtain $\lambda \in$ $(0.46,1.95)$ and for the $b \bar{b} \gamma \gamma$ mode, $\lambda \in(0.09,4.83)$, where the latter corresponds to the full interval, symmetric about the minimum. It is evident that the ratio provides a comparable exclusion region, especially considering the fact that ref. [38] considers relatively optimistic background subtraction. However, the ratio possesses advantages over the distribution analysis that may contain systematic uncertainties induced by the modelling of the shapes of both the signal and background. Note that an interesting study of the theoretical sensitivity of different initial states $\left(g g \rightarrow H H, q q^{\prime} \rightarrow H H q q^{\prime}, q \bar{q}^{\prime} \rightarrow W H H\right.$ and $q \bar{q} \rightarrow$ $\mathrm{ZHH}$ ) on the trilinear coupling can be found in [35]. 

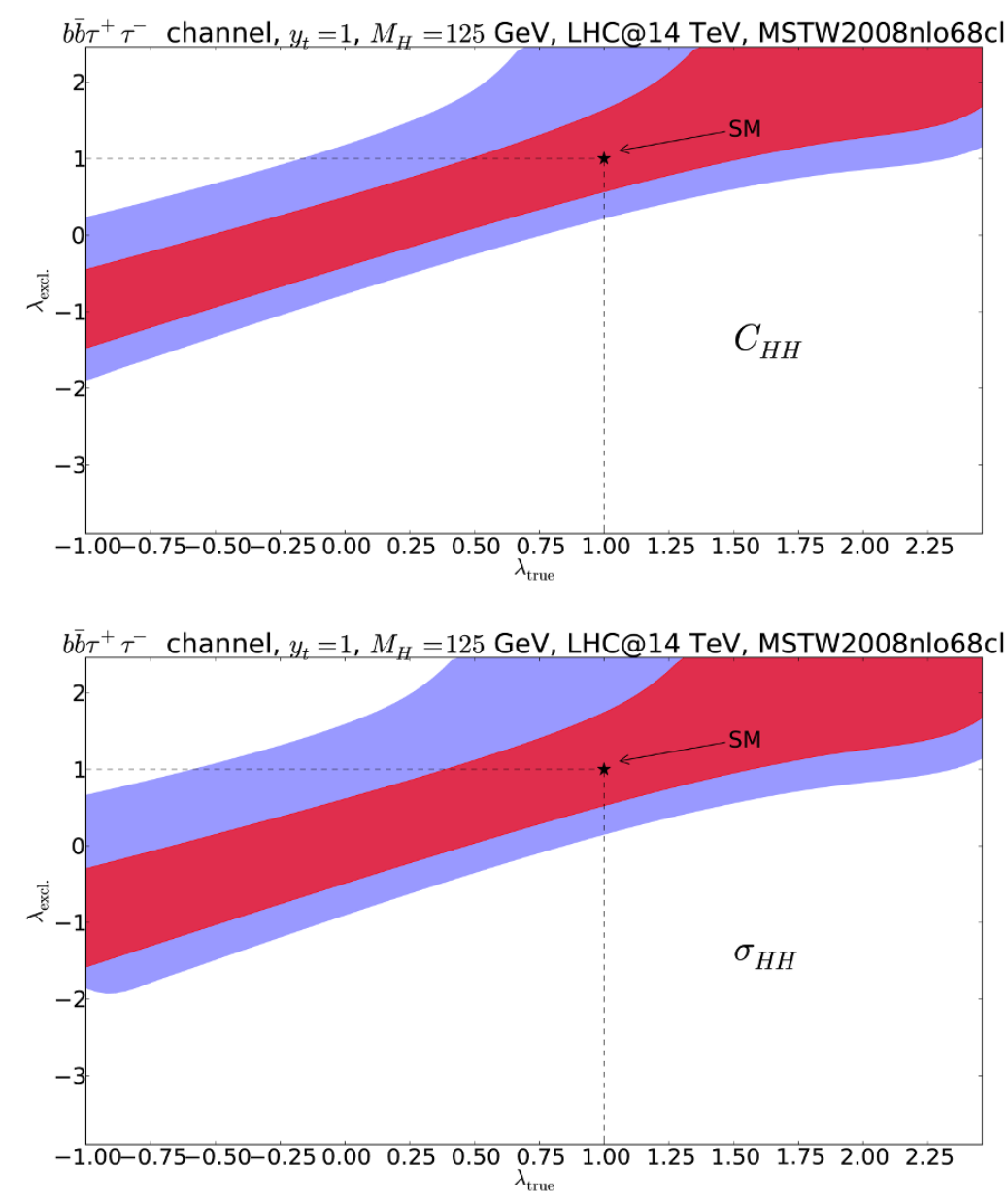

Figure 6. The expected exclusion for $\lambda$ in the simplified model we are considering, at one and two standard deviations for a given value of $\lambda_{\text {true }}$ at $600 \mathrm{fb}^{-1}$ for the $b \bar{b} \tau^{+} \tau^{-}$decay mode. The exclusion constructed from the ratio, $C_{H H}$, is shown on the top panel, whereas the exclusion obtained from the cross section, $\sigma_{H H}$, is shown on the bottom panel. We only show the region up to the symmetric minimum at $\lambda \simeq 2.46$.

Since the cross section for Higgs pair production, as well as the single Higgs cross section, both depend on the top coupling, a determination of $y_{t}$ and the triple coupling, $\lambda$, cannot be done independently through a measurement of the ratio $C_{H H} \cdot{ }^{11}$ The coupling $y_{t}$ can be deduced by observation of associated production of a single Higgs with top quark pairs [79] using boosted jet techniques that exploit the substructure of so-called 'fat' jets. ${ }^{12}$ Since the error on a determination of $y_{t}$ is expected to be $\mathcal{O}(15 \%)$ [68], an investigation of the possible constraints in the $y_{t}-\lambda$ plane is essential. This can be done for the Standard

\footnotetext{
${ }^{11}$ There exist many models in which the $H t \bar{t}$ coupling, $y_{t}$, can be changed, among other effects. See, for example, [54, 55, 72-78].

${ }^{12}$ Note that at the LHC no measurements of absolute couplings can be performed. It is however possible to make fits to Higgs couplings that are almost model-independent using weak theoretical assumptions. For further discussion see, for example, section 2 in ref. [68].
} 


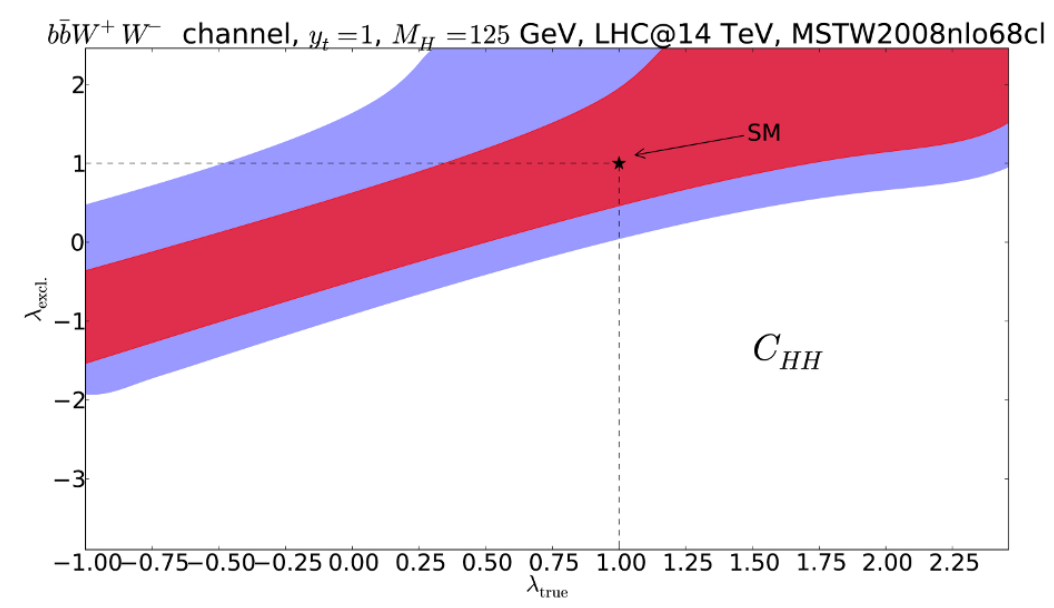

Figure 7. The expected exclusion in the simplified model we are considering, for $\lambda$ at one and two standard deviations for a given value of $\lambda_{\text {true }}$ at $600 \mathrm{fb}^{-1}$ for the $b \bar{b} W^{+} W^{-}$decay mode, constructed by using the ratio of cross sections $C_{H H}$. We only show the region up to the symmetric minimum at $\lambda \simeq 2.46$.

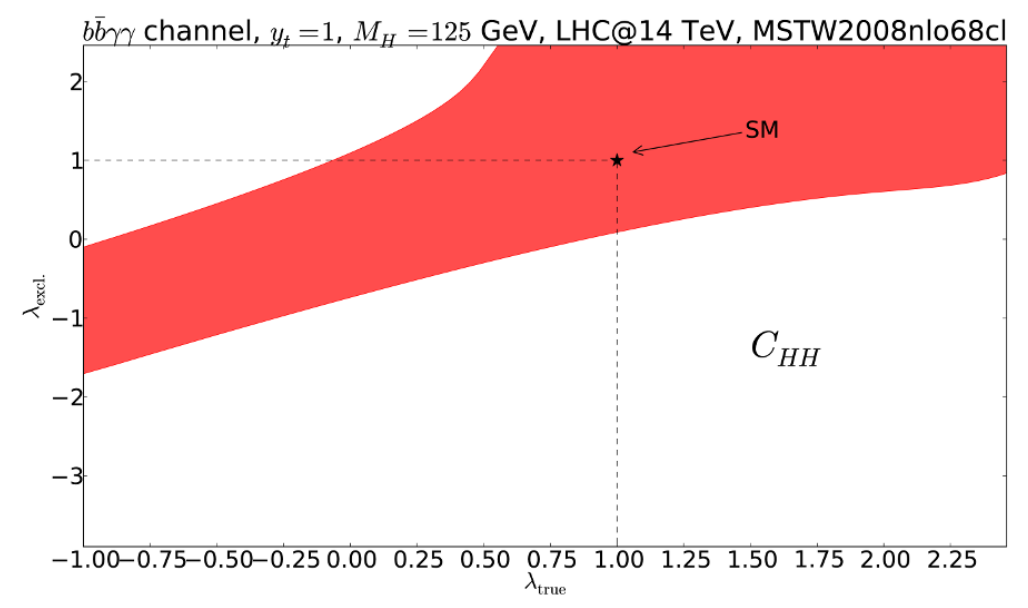

Figure 8. The expected exclusion in the simplified model we are considering, for $\lambda$ at one standard deviations for a given value of $\lambda_{\text {true }}$ at $600 \mathrm{fb}^{-1}$ for the $b \bar{b} \gamma \gamma$ decay mode, constructed by using the ratio of cross sections $C_{H H}$. The two standard deviations exclusion is not shown since it is weak. We only show the region up to the symmetric minimum at $\lambda \simeq 2.46$.

Model with the assumption $\lambda_{\text {true }}=1$ and $y_{t, \text { true }}=1$ in the simplified model. We can then calculate the induced error as we have done previously and calculate the $1 \sigma$ and $2 \sigma$ confidence levels on where the actual measurement will likely end up in the $y_{t}-\lambda$ plane. The results are shown in figures 9, 10 and 11 for $b \bar{b} \tau^{+} \tau^{-}, b \bar{b} W^{+} W^{-}$and $b \bar{b} \gamma \gamma$ respectively, given an integrated luminosity of $600 \mathrm{fb}^{-1}$. The figures illustrate an important point: for a model-independent determination of the Higgs triple self-coupling, a good measurement of $y_{t}$ is crucial. If, for example, we consider $y_{t}$ at the edges of the expected $\mathcal{O}(15 \%)$ error, then $y_{t}=0.85$ yields $\lambda \in(0.2,1.1)$ whereas $y_{t}=1.15$ yields $\lambda \in(1.1, \sim 2.4)$, using the 


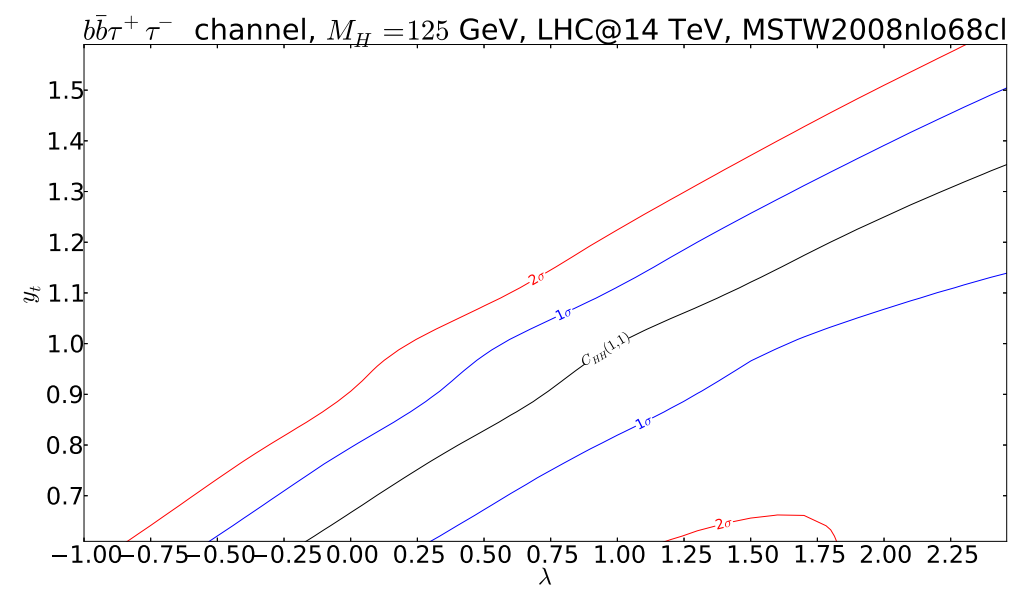

Figure 9. The $1 \sigma$ and $2 \sigma$ confidence regions in the $y_{t}-\lambda$ plane at $600 \mathrm{fb}^{-1}$ for the $b \bar{b} \tau^{+} \tau^{-}$decay mode, derived using $C_{H H}$, within the SM $\left(\lambda_{\text {true }}=1\right.$ and $\left.y_{t, \text { true }}=1\right)$.

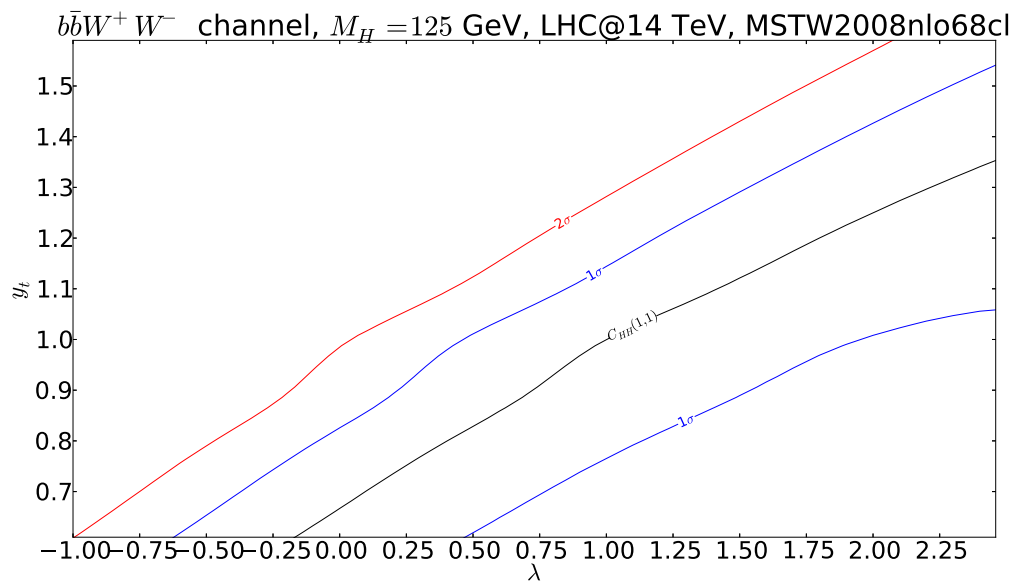

Figure 10. The $1 \sigma$ and $2 \sigma$ confidence regions in the $y_{t}-\lambda$ plane at $600 \mathrm{fb}^{-1}$ for the $b \bar{b} W^{+} W^{-}$ decay mode, derived using $C_{H H}$, within the SM $\left(\lambda_{\text {true }}=1\right.$ and $\left.y_{t, \text { true }}=1\right)$. In the lower-right corner the exclusion is weak and only the one standard deviation curve is shown.

$b \bar{b} \tau^{+} \tau^{-}$channel (Figure 9), both at $1 \sigma$. This is a result of the sensitivity of the single and double cross sections on $y_{t}$ (see eq. (2.3)).

\section{Conclusions}

We have considered the theoretical error on the ratio of cross sections of double-to-single Higgs production, $C_{H H}$, at a $14 \mathrm{TeV} \mathrm{LHC}$, including scale variation and parton density function uncertainties. Under the assumption that the double and single Higgs boson production cross sections possess a similar form of higher-order corrections, which we motivated in section 3 , we showed in the same section that the ratio is a more theoretically stable quantity than the cross section itself. Subsequently, assuming a $5 \%$ total theoretical error 


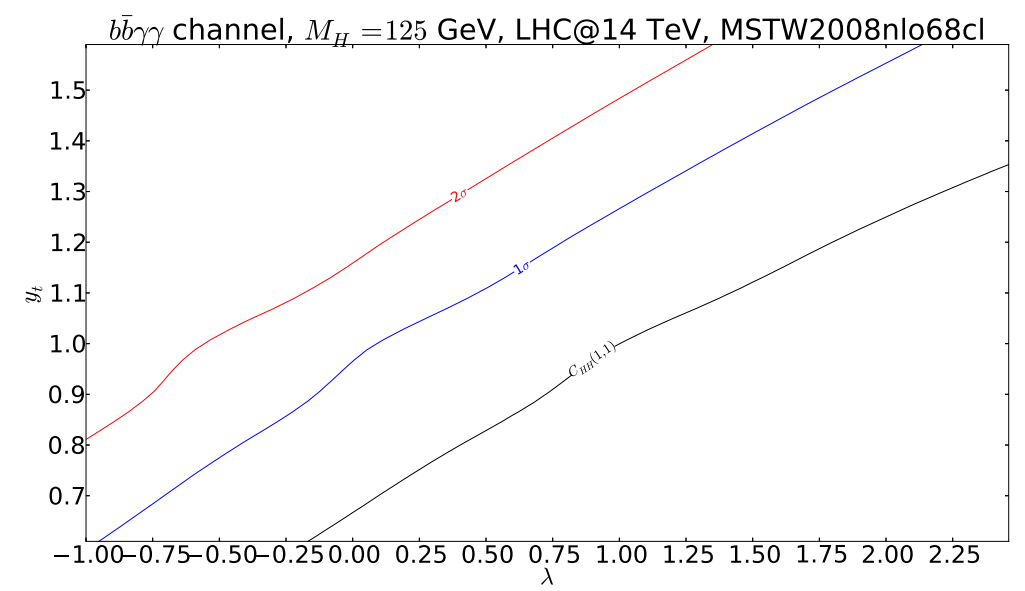

Figure 11. The $1 \sigma$ and $2 \sigma$ confidence regions in the $y_{t}-\lambda$ plane at $600 \mathrm{fb}^{-1}$ for the $b \bar{b} \gamma \gamma$ decay mode, derived using $C_{H H}$, within the SM $\left(\lambda_{\text {true }}=1\right.$ and $\left.y_{t, \text { true }}=1\right)$. In the lower-right corner the exclusion is very weak and hence the one and two standard deviation curves are off the scale of the figure.

on $C_{H H}$, and using conservative assumptions on the experimental uncertainties of the quantities involved in measuring the ratio, we used this ratio to construct possible exclusions in a set of simplified models, given a true value of the corresponding Higgs self-coupling parameter, at a $14 \mathrm{TeV}$ LHC and integrated luminosities of $600 \mathrm{fb}^{-1}$ and $3000 \mathrm{fb}^{-1}$. Within the Standard Model we concluded that it is possible to constrain the trilinear coupling to be positive, at $95 \%$ confidence level at $600 \mathrm{fb}^{-1}$, only using the discovery of the three viable channels. We also showed that a naive combination of the 'uncertainties' at $1 \sigma$ over the three channels indicates that a measurement of accuracy $\sim+30 \%$ and $\sim-20 \%$ is possible simply by using the ratio $C_{H H}$ at $3000 \mathrm{fb}^{-1}$. The present work outlines the most precise method of determination of the Higgs triple self-coupling in the SM to date. We have also considered the uncertainty on the top-Higgs coupling and have constructed the possible exclusion region in the $y_{t}-\lambda$ plane. Thus, we concluded that an accurate determination of the $H t \bar{t}$ coupling, $y_{t}$, is crucial to the determination of the Higgs boson triple self-coupling.

It is evident that deviations from expected exclusions within the SM would be an indication of some inconsistency in these assumptions that would require further assessment in the form of new physics models. Given the framework that we have outlined in the present paper, the parameter space relevant to Higgs pair production can be probed using the ratio $C_{H H}$ in any BSM theory. Furthermore, it is obvious from the present study, as well as previous ones, that the measurement of the Higgs boson trilinear self-coupling is a challenging task, and further effort, both on behalf of theorists and experimentalists, should be made in order to obtain the best possible measurement during the lifetime of the LHC. 


\section{Acknowledgments}

We would like to thank Elisabetta Furlan, Thomas Gehrmann, Massimiliano Grazzini, Paolo Torrielli and Graeme Watt for interesting and useful discussions. We would also like to thank the Referee for his helpful comments and suggestions. This work was supported by the Swiss National Science Foundation under contracts 200020-138206 and 200020141360/1 (AP) and 200020-126632 (FG) and by the Research Executive Agency of the European Union under the Grant Agreement number PITN-GA-2010-264564 (LHCPhenoNet). JZ is supported by the ERC Advanced Grant EFT4LHC of the European Research Council, the Cluster of Excellence Precision Physics, Fundamental Interactions and Structure of Matter (PRISMA-EXC 1098).

\section{References}

[1] ATLAS collaboration, Observation of a new particle in the search for the standard model Higgs boson with the ATLAS detector at the LHC, Phys. Lett. B 716 (2012) 1 [arXiv: 1207.7214] [INSPIRE].

[2] CMS collaboration, Observation of a new boson at a mass of $125 \mathrm{GeV}$ with the CMS experiment at the LHC, CMS-HIG-12-028 (2012).

[3] CMS collaboration, Combination of standard model Higgs boson searches and measurements of the properties of the new boson with a mass near $125 \mathrm{GeV}$, CMS-PAS-HIG-12-045 (2012).

[4] ATLAS collaboration, Coupling properties of the new Higgs-like boson observed with the ATLAS detector at the LHC, ATLAS-CONF-2012-127 (2012).

[5] ATLAS collaboration, An update of combined measurements of the new Higgs-like boson with high mass resolution channels, ATLAS-CONF-2012-170 (2012).

[6] J. Ellis and T. You, Global analysis of the Higgs candidate with mass $~ 125 \mathrm{GeV}$, JHEP 09 (2012) 123 [arXiv: 1207.1693] [INSPIRE].

[7] D. Carmi, A. Falkowski, E. Kuflik and T. Volansky, Interpreting LHC Higgs results from natural new physics perspective, JHEP 07 (2012) 136 [arXiv:1202.3144] [INSPIRE].

[8] A. Azatov, R. Contino and J. Galloway, Model-independent bounds on a light Higgs, JHEP 04 (2012) 127 [Erratum ibid. 1304 (2013) 140] [arXiv: 1202.3415] [INSPIRE].

[9] J. Espinosa, C. Grojean, M. Muhlleitner and M. Trott, Fingerprinting Higgs suspects at the LHC, JHEP 05 (2012) 097 [arXiv: 1202.3697] [INSPIRE].

[10] P.P. Giardino, K. Kannike, M. Raidal and A. Strumia, Reconstructing Higgs boson properties from the LHC and Tevatron data, JHEP 06 (2012) 117 [arXiv:1203.4254] [INSPIRE].

[11] A. Azatov, R. Contino, D. Del Re, J. Galloway, M. Grassi et al., Determining Higgs couplings with a model-independent analysis of $H \rightarrow \gamma \gamma$, JHEP 06 (2012) 134 [arXiv: 1204.4817] [INSPIRE].

[12] M. Klute, R. Lafaye, T. Plehn, M. Rauch and D. Zerwas, Measuring Higgs couplings from LHC data, Phys. Rev. Lett. 109 (2012) 101801 [arXiv: 1205. 2699] [INSPIRE].

[13] J.R. Espinosa, M. Muhlleitner, C. Grojean and M. Trott, Probing for invisible Higgs decays with global fits, JHEP 09 (2012) 126 [arXiv:1205.6790] [INSPIRE]. 
[14] D. Carmi, A. Falkowski, E. Kuflik and T. Volansky, Interpreting the Higgs, arXiv:1206.4201 [INSPIRE].

[15] J. Chang, K. Cheung, P.-Y. Tseng and T.-C. Yuan, Distinguishing various models of the $125 \mathrm{GeV}$ boson in vector boson fusion, JHEP 12 (2012) 058 [arXiv:1206.5853] [INSPIRE].

[16] S. Chang, C.A. Newby, N. Raj and C. Wanotayaroj, Revisiting theories with enhanced Higgs couplings to weak gauge bosons, Phys. Rev. D 86 (2012) 095015 [arXiv:1207.0493] [INSPIRE].

[17] I. Low, J. Lykken and G. Shaughnessy, Have we observed the Higgs (imposter)?, Phys. Rev. D 86 (2012) 093012 [arXiv:1207.1093] [INSPIRE].

[18] T. Corbett, O. Eboli, J. Gonzalez-Fraile and M. Gonzalez-Garcia, Constraining anomalous Higgs interactions, Phys. Rev. D 86 (2012) 075013 [arXiv: 1207.1344] [INSPIRE].

[19] P.P. Giardino, K. Kannike, M. Raidal and A. Strumia, Is the resonance at $125 \mathrm{GeV}$ the Higgs boson?, Phys. Lett. B 718 (2012) 469 [arXiv:1207.1347] [INSPIRE].

[20] M. Montull and F. Riva, Higgs discovery: the beginning or the end of natural EWSB?, JHEP 11 (2012) 018 [arXiv:1207.1716] [INSPIRE].

[21] J. Espinosa, C. Grojean, M. Muhlleitner and M. Trott, First glimpses at Higgs' face, JHEP 12 (2012) 045 [arXiv: 1207.1717] [INSPIRE].

[22] D. Carmi, A. Falkowski, E. Kuflik, T. Volansky and J. Zupan, Higgs after the discovery: a status report, JHEP 10 (2012) 196 [arXiv:1207.1718] [INSPIRE].

[23] S. Banerjee, S. Mukhopadhyay and B. Mukhopadhyaya, New Higgs interactions and recent data from the LHC and the Tevatron, JHEP 10 (2012) 062 [arXiv:1207.3588] [INSPIRE].

[24] F. Bonnet, T. Ota, M. Rauch and W. Winter, Interpretation of precision tests in the Higgs sector in terms of physics beyond the standard model, Phys. Rev. D 86 (2012) 093014 [arXiv: 1207.4599] [INSPIRE].

[25] T. Plehn and M. Rauch, Higgs couplings after the discovery, Europhys. Lett. 100 (2012) 11002 [arXiv: 1207.6108] [INSPIRE].

[26] A. Djouadi, Precision Higgs coupling measurements at the LHC through ratios of production cross sections, arXiv:1208.3436 [INSPIRE].

[27] G. Cacciapaglia, A. Deandrea, G.D. La Rochelle and J.-B. Flament, Higgs couplings beyond the standard model, JHEP 03 (2013) 029 [arXiv: 1210.8120] [INSPIRE].

[28] E. Masso and V. Sanz, Limits on anomalous couplings of the Higgs to electroweak gauge bosons from LEP and LHC, Phys. Rev. D 87 (2013) 033001 [arXiv:1211.1320] [InSPIRE].

[29] R.S. Gupta, M. Montull and F. Riva, SUSY faces its Higgs couplings, JHEP 04 (2013) 132 [arXiv: 1212.5240] [INSPIRE].

[30] G. Bélanger, B. Dumont, U. Ellwanger, J. Gunion and S. Kraml, Higgs couplings at the end of 2012, JHEP 02 (2013) 053 [arXiv: 1212 .5244] [INSPIRE].

[31] U. Baur, T. Plehn and D.L. Rainwater, Measuring the Higgs boson self coupling at the LHC and finite top mass matrix elements, Phys. Rev. Lett. 89 (2002) 151801 [hep-ph/0206024] [INSPIRE].

[32] U. Baur, T. Plehn and D.L. Rainwater, Determining the Higgs boson selfcoupling at hadron colliders, Phys. Rev. D 67 (2003) 033003 [hep-ph/0211224] [INSPIRE]. 
[33] M.J. Dolan, C. Englert and M. Spannowsky, Higgs self-coupling measurements at the LHC, JHEP 10 (2012) 112 [arXiv:1206.5001] [INSPIRE].

[34] A. Papaefstathiou, L.L. Yang and J. Zurita, Higgs boson pair production at the LHC in the $b \bar{b} W^{+} W^{-}$channel, Phys. Rev. D 87 (2013) 011301 [arXiv:1209.1489] [INSPIRE].

[35] J. Baglio, A. Djouadi, R. Gröber, M. Mühlleitner, J. Quevillon et al., The measurement of the Higgs self-coupling at the LHC: theoretical status, JHEP 04 (2013) 151 [arXiv: 1212.5581] [INSPIRE].

[36] A. Djouadi, W. Kilian, M. Muhlleitner and P. Zerwas, Production of neutral Higgs boson pairs at LHC, Eur. Phys. J. C 10 (1999) 45 [hep-ph/9904287] [InSPIRE].

[37] T. Plehn and M. Rauch, The quartic Higgs coupling at hadron colliders, Phys. Rev. D 72 (2005) 053008 [hep-ph/0507321] [INSPIRE].

[38] U. Baur, T. Plehn and D.L. Rainwater, Probing the Higgs selfcoupling at hadron colliders using rare decays, Phys. Rev. D 69 (2004) 053004 [hep-ph/0310056] [INSPIRE].

[39] J.M. Butterworth, A.R. Davison, M. Rubin and G.P. Salam, Jet substructure as a new Higgs search channel at the LHC, Phys. Rev. Lett. 100 (2008) 242001 [arXiv:0802.2470] [INSPIRE].

[40] A. Abdesselam, E.B. Kuutmann, U. Bitenc, G. Brooijmans, J. Butterworth et al., Boosted objects: a probe of beyond the standard model physics, Eur. Phys. J. C 71 (2011) 1661 [arXiv: 1012.5412] [INSPIRE].

[41] T. Plehn, M. Spira and P. Zerwas, Pair production of neutral Higgs particles in gluon-gluon collisions, Nucl. Phys. B 479 (1996) 46 [Erratum ibid. B 531 (1998) 655] [hep-ph/9603205] [INSPIRE].

[42] S. Dawson, S. Dittmaier and M. Spira, Neutral Higgs boson pair production at hadron colliders: QCD corrections, Phys. Rev. D 58 (1998) 115012 [hep-ph/9805244] [INSPIRE].

[43] F. Gianotti, M. Mangano, T. Virdee, S. Abdullin, G. Azuelos et al., Physics potential and experimental challenges of the LHC luminosity upgrade, Eur. Phys. J. C 39 (2005) 293 [hep-ph/0204087] [INSPIRE].

[44] M. Moretti, S. Moretti, F. Piccinini, R. Pittau and A. Polosa, Higgs boson self-couplings at the LHC as a probe of extended Higgs sectors, JHEP 02 (2005) 024 [hep-ph/0410334] [INSPIRE].

[45] E.N. Glover and J. van der Bij, Higgs boson pair production via gluon fusion, Nucl. Phys. B 309 (1988) 282 [INSPIRE].

[46] M. Spira, hpair, http://people.web.psi.ch/spira/hpair/.

[47] A. Martin, W. Stirling, R. Thorne and G. Watt, Parton distributions for the LHC, Eur. Phys. J. C 63 (2009) 189 [arXiv:0901.0002] [INSPIRE].

[48] R. Grober and M. Muhlleitner, Composite Higgs boson pair production at the LHC, JHEP 06 (2011) 020 [arXiv: 1012.1562] [INSPIRE].

[49] R. Contino, M. Ghezzi, M. Moretti, G. Panico, F. Piccinini et al., Anomalous couplings in double Higgs production, JHEP 08 (2012) 154 [arXiv:1205.5444] [INSPIRE].

[50] G.D. Kribs and A. Martin, Enhanced di-Higgs production through light colored scalars, Phys. Rev. D 86 (2012) 095023 [arXiv: 1207.4496] [InSPIRE]. 
[51] M. Gillioz, R. Grober, C. Grojean, M. Muhlleitner and E. Salvioni, Higgs low-energy theorem (and its corrections) in composite models, JHEP 10 (2012) 004 [arXiv:1206.7120] [INSPIRE].

[52] S. Dawson, E. Furlan and I. Lewis, Unravelling an extended quark sector through multiple Higgs production?, Phys. Rev. D 87 (2013) 014007 [arXiv: 1210.6663] [INSPIRE].

[53] M.J. Dolan, C. Englert and M. Spannowsky, New physics in LHC Higgs boson pair production, Phys. Rev. D 87 (2013) 055002 [arXiv:1210.8166] [InSPIRE].

[54] B. Patt and F. Wilczek, Higgs-field portal into hidden sectors, hep-ph/0605188 [INSPIRE].

[55] R. Schabinger and J.D. Wells, A minimal spontaneously broken hidden sector and its impact on Higgs boson physics at the large hadron collider, Phys. Rev. D 72 (2005) 093007 [hep-ph/0509209] [INSPIRE].

[56] G. Branco, P. Ferreira, L. Lavoura, M. Rebelo, M. Sher et al., Theory and phenomenology of two-Higgs-doublet models, Phys. Rept. 516 (2012) 1 [arXiv:1106.0034] [INSPIRE].

[57] R.S. Gupta, H. Rzehak and J.D. Wells, How well do we need to measure Higgs boson couplings?, Phys. Rev. D 86 (2012) 095001 [arXiv:1206.3560] [INSPIRE].

[58] M.L. Mangano and J. Rojo, Cross section ratios between different CM energies at the LHC: opportunities for precision measurements and BSM sensitivity, JHEP 08 (2012) 010 [arXiv: 1206.3557] [INSPIRE].

[59] T. Binoth, S. Karg, N. Kauer and R. Ruckl, Multi-Higgs boson production in the standard model and beyond, Phys. Rev. D 74 (2006) 113008 [hep-ph/0608057] [INSPIRE].

[60] M. Spira, HIGLU: a program for the calculation of the total Higgs production cross-section at hadron colliders via gluon fusion including QCD corrections, hep-ph/9510347 [INSPIRE].

[61] G. Watt, Parton distribution function dependence of benchmark standard model total cross sections at the $7 \mathrm{TeV}$ LHC, JHEP 09 (2011) 069 [arXiv:1106.5788] [INSPIRE].

[62] D.Y. Shao, C.S. Li, H.T. Li and J. Wang, Threshold resummation effects in Higgs boson pair production at the LHC, arXiv:1301.1245 [INSPIRE].

[63] D. de Florian and M. Grazzini, Higgs production through gluon fusion: updated cross sections at the Tevatron and the LHC, Phys. Lett. B 674 (2009) 291 [arXiv:0901.2427] [INSPIRE].

[64] V. Ahrens, T. Becher, M. Neubert and L.L. Yang, Renormalization-group improved prediction for Higgs production at hadron colliders, Eur. Phys. J. C 62 (2009) 333 [arXiv: 0809.4283] [INSPIRE].

[65] D. de Florian, G. Ferrera, M. Grazzini and D. Tommasini, Higgs boson production at the LHC: transverse momentum resummation effects in the $H \rightarrow 2 \gamma, H \rightarrow W W \rightarrow$ lnulv and $H \rightarrow Z Z \rightarrow 4 l$ decay modes, JHEP 06 (2012) 132 [arXiv:1203.6321] [INSPIRE].

[66] LhC Higgs Cross section Working Group collaboration, S. Dittmaier et al., Handbook of LHC Higgs cross sections: 1. Inclusive observables, arXiv:1101.0593 [INSPIRE].

[67] R.D. Ball, S. Carrazza, L. Del Debbio, S. Forte, J. Gao et al., Parton distribution benchmarking with LHC data, JHEP 04 (2013) 125 [arXiv: 1211.5142] [INSPIRE].

[68] M.E. Peskin, Comparison of LHC and ILC capabilities for Higgs boson coupling measurements, arXiv:1207.2516 [INSPIRE]. 
[69] M. Farina, C. Grojean, F. Maltoni, E. Salvioni and A. Thamm, Lifting degeneracies in Higgs couplings using single top production in association with a Higgs boson, JHEP 05 (2013) 022 [arXiv: 1211.3736] [INSPIRE].

[70] S. Biswas, E. Gabrielli and B. Mele, Single top and Higgs associated production as a probe of the Htt coupling sign at the LHC, JHEP 01 (2013) 088 [arXiv:1211.0499] [INSPIRE].

[71] G.Landsberg et al., CMS at the High Energy Frontier. Contribution to the Update of the European Strategy for Particle Physics, https : //indico. cern. ch/contributionDisplay . py? contribId=144\&conf Id=175067 (2012)

[72] K. Agashe, G. Perez and A. Soni, Collider signals of top quark flavor violation from a warped extra dimension, Phys. Rev. D 75 (2007) 015002 [hep-ph/0606293] [INSPIRE].

[73] S. Casagrande, F. Goertz, U. Haisch, M. Neubert and T. Pfoh, Flavor physics in the Randall-Sundrum model: I. Theoretical setup and electroweak precision tests, JHEP 10 (2008) 094 [arXiv: 0807.4937] [INSPIRE].

[74] K. Agashe and R. Contino, Composite Higgs-Mediated FCNC, Phys. Rev. D 80 (2009) 075016 [arXiv: 0906.1542] [INSPIRE].

[75] A. Djouadi and G. Moreau, Higgs production at the LHC in warped extra-dimensional models, Phys. Lett. B 660 (2008) 67 [arXiv:0707.3800] [INSPIRE].

[76] A. Falkowski, Pseudo-Goldstone Higgs production via gluon fusion, Phys. Rev. D 77 (2008) 055018 [arXiv: 0711.0828] [INSPIRE].

[77] A. Azatov, M. Toharia and L. Zhu, Higgs mediated FCNC's in warped extra dimensions, Phys. Rev. D 80 (2009) 035016 [arXiv:0906.1990] [InSPIRE].

[78] S. Casagrande, F. Goertz, U. Haisch, M. Neubert and T. Pfoh, The custodial Randall-Sundrum model: from precision tests to Higgs physics, JHEP 09 (2010) 014 [arXiv: 1005.4315] [INSPIRE].

[79] T. Plehn, G.P. Salam and M. Spannowsky, Fat jets for a light Higgs, Phys. Rev. Lett. 104 (2010) 111801 [arXiv:0910.5472] [INSPIRE]. 